\title{
Les Sphingidae de forêt en Guyane française: Comparaison des populations en forêt inondable et non inondable
}

\author{
Sphingidae in French Guiana's primary rainforest: \\ Comparison of Sphingidae populations between flooded \\ and no flooded rainforest
}

\author{
Philippe Cerdan, Véronique Horeau, Sandrine Richard \\ Laboratoire Environnement de Petit Saut, EDF-CNEH B.P. 823, 97388 Kourou cedex, Guyane \\ française
}

Résumé. - Dans le cadre des études menées sur l'environnement du barrage hydroélectrique à Petit Saut, un suivi des populations de Sphingidae se poursuit depuis 1990. Deux stations ont été particulièrement prospectées: Petit Saut, caractéristique d'une forêt primaire de basse altitude recouvrant la majeure partie du plateau des Guyanes et Saut Dalles, zone de forêt inondée à marécage arbustif. Les différents tests montrent que la zone marécageuse de Saut Dalles est moins riche quantitativement et qualitativement que la zone non inondable de Petit Saut. II n'existe pas d'espèces caractéristiques de la zone inondable. L'ensemble des analyses montre que, même en milieu tropical l'étude des peuplements doit être réalisée au moins sur une année complète.

Mots-clés. - Lepidoptères, Sphingidae, Guyane, forêt primaire, zone marécageuse.

Summary. - As part of complementary studies into the surrounding environment of the Sinnamary's drainage basin following the construction of hydroelectric dam, a continued research program into Sphingidae populations has been going on for the past three years.

Two areas have been studied in particular:

- Petit Saut - characteristic of the low altitude primary rainforest which covers the most part of Guyana's table-lands;

- Saut Dalles - area of flooded forest with shrub filled marsh lands.

The different tests reveal that the marsh tracts are less rich both quantitatively and qualitatively. There are no particular characteristic species in these areas. Overall, the various studies have shown that research into Sphingidae populations in tropical zones must be carried out for at least one full year.

Key words. - Lepidoptera, Sphingidae, French Guiana, primary rainforest, marsh tract lands. 


\section{INTRODUCTION}

Depuis 1990, en Guyane à Petit Saut, EDF construit un barrage en milieu équatorial, sur le fleuve Sinnamary. Lors de la mise en eau en 1994, la retenue va entraîner la disparition de $310 \mathrm{~km}^{2}$ de forêt. Depuis 1983 des études sur l'environnement sont menées par plusieurs organismes de recherche. Un laboratoire a été construit sur le site en 1991.

Les forêts tropicales sont caractérisées par une biodiversité très importante, qui atteint une ampleur considérable chez les insectes.

L'homogénéité des peuplements faunistiques ou floristiques de cette forêt n'est qu'apparente. Divers écosystèmes se cotoient, en particulier des zones de forêt jamais inondées et des zones de forêt inondées en saison des pluies. Dans le cadre d'une étude menée sur les Sphingidae, il nous a paru intéressant de comparer leurs populations dans ces deux zones. Nous avons choisi de travailler sur Petit Saut, zone de forêt primaire non inondable et sur Saut Dalles, zone de forêt inondée et de marécage arbustif (photo 1).

Les Sphingidae représentent une famille maintenant bien connue en Guyane, principalement grâce aux travaux d'Haxaire (Haxaire 1985, 1986, 1987, 1988, 1990; Haxaire et al. 1986, 1987), plus récemment nous avons encore recensé des espèces nouvelles pour la Guyane, (Cerdan, 1992).
Dans la pollinisation des fleurs, les espèces de cette famille jouent un rôle comparable à celui des chauvesouris (Eisikowitch et al., 1971; Kislev et al., 1972; Linhart et al., 1977; Miller, 1981; Haber et al., 1989...). Elles sont régulièrement consommées par les poissons (photo 2) et certaines espèces sont également des bioindicateurs (Cerdan, 1991, 1992).

Dans cette étude nous avons également voulu souligner la nécessité du nombre important de nuits de piégeage réalisées sur plusieurs saisons.

\section{MÉTHODES}

La méthode de capture par piégeage lumineux a été retenue pour cette étude. Cette méthode est efficace pour l'échantillonnage de nombreux insectes aériens ayant une activité nocturne et crépusculaire (Tshernyshev et al., 1973; Blomberg et al., 1978; Fontaine, 1982; Usseglio-Polatera et al., 1987...). Sauf pour quelques espèces (Haxaire, 1992), elle est particulièrement efficace pour l'étude des Sphingidae. Le piégeage est réalisé avec des lampes à décharge de 125, 250 et 700 Watts. Dix campagnes de prélèvements ont été effectuées, 5 dans chaque station : en décembre 1990, juin, août et novembre 1991 et en janvier 1992. Ces prélèvements comportent chacun, trois nuits complètes de piégeage lumineux (18 h à $7 \mathrm{~h}$ ). Les piégeages sont effectués en "lune noire" (absence de 


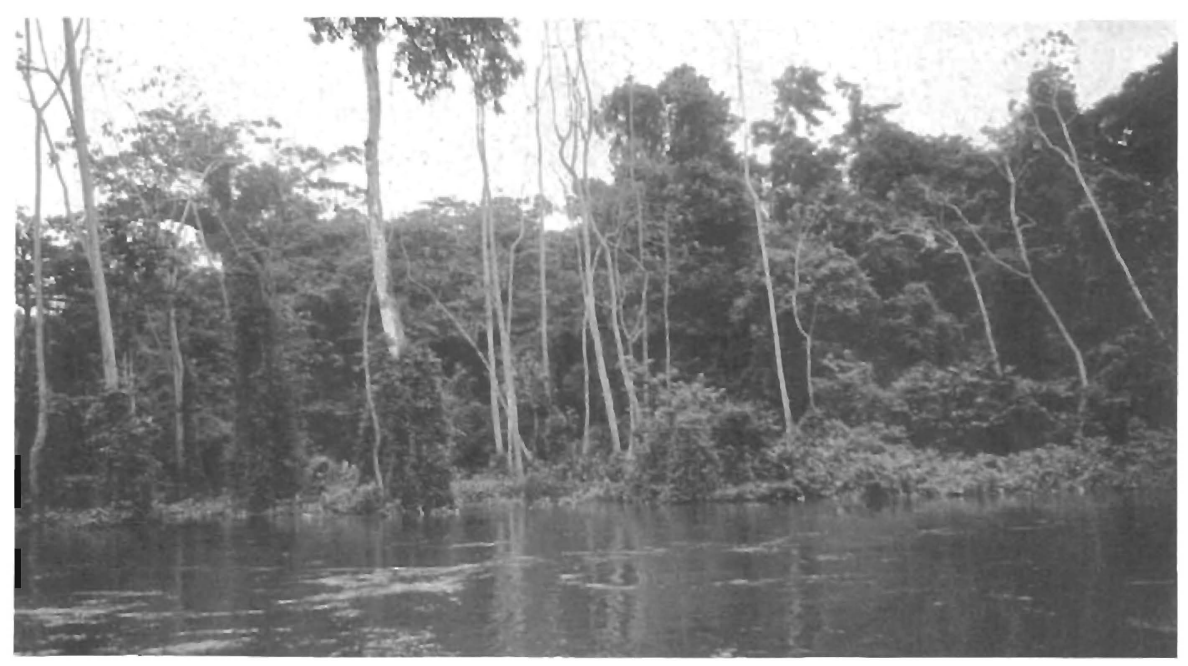

Photo 1. - Saut Dalles : zone de forêt inondée et de marécage arbustif. Photo 1. - Saut Dalles: flooded forest area with shrub filled marsh land.

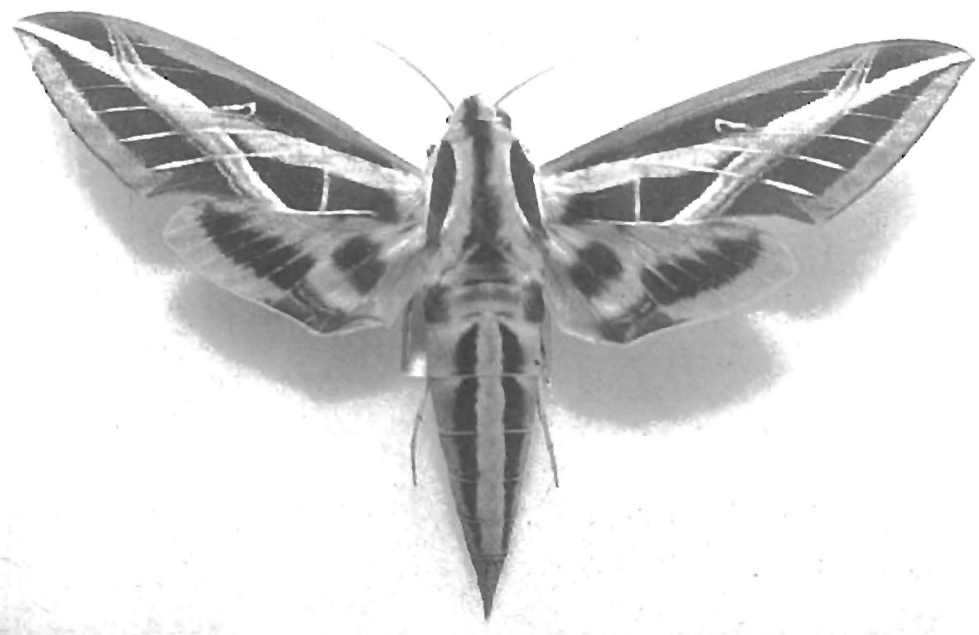

Photo 2. - Exemple de Sphingidae (Eumorpha fasciata) trouvé dans un estomac de poisson. Photo 2. - Sphingidae sample (Eumorpha fasciata) discovered in fish stomach. 
lune) pour une meilleure efficacité du piégeage.

Tous les individus sont comptés puis, soit marqués et relâchés (légère entaille sur une aile), soit conservés.

L'indice de Shannon et l'équitabilité sont calculés pour ces deux stations.

Différents tests sont réalisés pour chacune des cinq campagnes de prélèvement. (test $t$, Wilcoxon - Mann Whitney, Khi carré).

\section{RÉSULTATS}

Indice de Shannon :

Pour les 2 stations, les indices de Shannon montrent que les peuple- ments sont riches et stables, proches de l'indice de Shannon maximum. II en est de même pour l'équitabilité dont la valeur la plus faible est de 0,83 (tableaux 1 et 2).

Nombre d'espèces:

Lors des 5 piégeages, 47 espèces ont été capturées à Saut Dalles et 69 à Petit Saut (tableau 3).

Toutes les espèces présentes à Saut Dalles sont également présentes à Petit Saut à l'exception de Xylophanes ceratomoides. II s'agit d'une espece rare en Guyane (Haxaire, 1985, 1986). Nous l'avons cependant déjà récoltée à Petit Saut en dehors des mois étudiés.

Tableaux 1 et 2. - Indices de Shannon et d'équitabilité.

Tables 1 and 2. - Shannon and equitability indexes.

Petit Saut

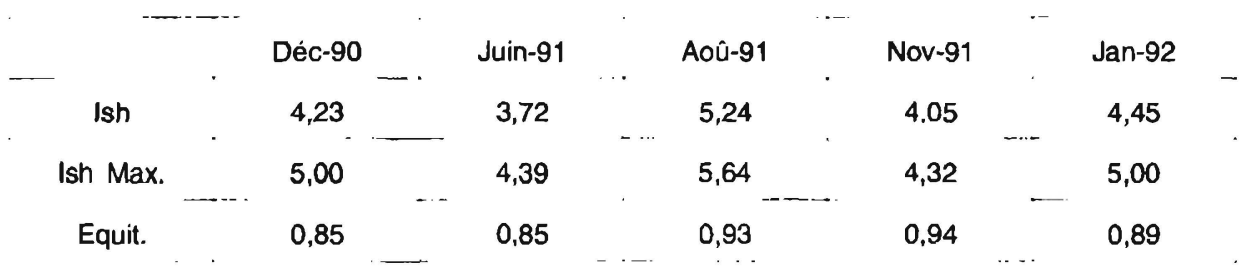

Saut Dalles

\begin{tabular}{|c|c|c|c|c|c|}
\hline & Déc-90 & Jui-91 & Aoû-91 & Nov-91 & Jan-92 \\
\hline Ish & 3.76 & 3,93 & 3,68 & 2.87 & 4.61 \\
\hline Ish Max. & 4,09 & 4.39 & 4,46 & 3,00 & 5,13 \\
\hline Equit. & 0,92 & 0,89 & 0,83 & 0.96 & 0,90 \\
\hline
\end{tabular}


Tableau 3. - Espèces récoltées à Petit Saut et à Saut Dalles lors des 5 prélèvements. Table 3. - Collected species in Petit Saut and Saut Dalles (5 samplings)

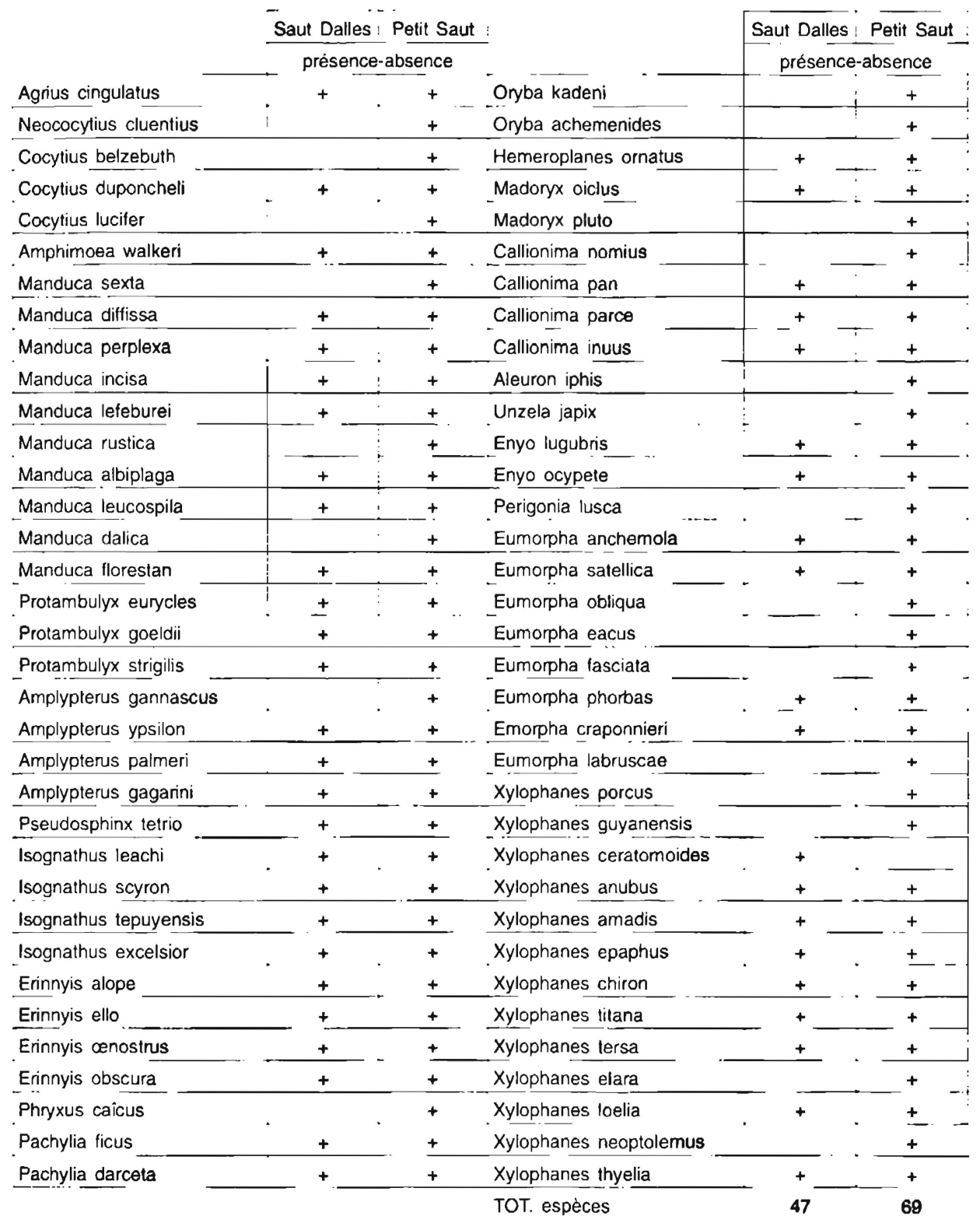


Tableau 4. - Comparaison du nombre d'espèces capturées à Saut Dalles et à Petit Saut.

Table 4. - Comparison between traped species number in Saut Dalles and Petit Saut.

\begin{tabular}{|c|c|c|c|c|c|c|}
\hline & $\begin{array}{l}\text { Décembre } \\
1990\end{array}$ & $\begin{array}{l}\text { Juin } \\
1991\end{array}$ & $\begin{array}{l}\text { Août } \\
1991\end{array}$ & $\begin{array}{c}\text { Novembre } \\
1991\end{array}$ & $\begin{array}{l}\text { Janvier } \\
1992\end{array}$ & $\begin{array}{c}\text { Ensemble } \\
\text { des prélèvements }\end{array}$ \\
\hline Petit Saut & 32 & 21 & 50 & 20 & 32 & 69 \\
\hline Saut Dalles & 17 & 21 & 22 & 8 & 35 & 47 \\
\hline \multirow[t]{2}{*}{$\mathrm{x} 2$} & 5,83 & 0 & 15,85 & 5,85 & 0,19 & 8,42 \\
\hline & Sign & Non Sig & $\mathrm{Ht}$ Sign & Sign & on Sign & Ht Sign \\
\hline
\end{tabular}

L'étude des 5 prélèvements montre que les différences ne sont pas significatives pour les mois de juin 1991 et janvier 1992. A l'inverse, pour les mois de décembre 1990, août et novembre 1991, ces différences sont significatives ou hautement significatives. (Test de Khi carré).

$\chi^{2}=(\alpha=0,05)=3,84$

$\hat{\chi}^{2}=(\alpha=0,01)=6,64$

Pour l'ensemble des cinq prélèvements (47 espèces à Saut Dalles et 69 à Petit Saut) la différence est hautement significative (tableau 4).

La comparaison du nombre d'individus capturés (tableau 5), montre une différence hautement significative entre Saut Dalles et Petit Saut (test $t$ et test Wilcoxon - Mann - Whitney).

Importance du nombre de prélèvements :

Les figures 1 et 2 mettent en évidence l'utilité d'une série de piégeages d'un minimum de trois nuits. Si dans 9 prèlèvements, la majorité des espèces est capturée lors des deux premières nuits et moins de 4 espèces supplémentaires lors de la troisième nuit, à l'inverse durant le piégeage de Petit Saut d'août 1990, 10 espèces nouvelles ont été récoltées lors de la troisième nuit. Ce résultat est cependant à mettre en parallèle avec le nombre d'individus important capturé ce mois ci.

Dans chaque station, la figure 3 montre que 5 séries de piégeages représentent un échantillon minimum. En effet 6 espèces nouvelles ont été récupérées à Petit Saut comme à Saut Dalles lors des 3 dernières nuits du cinquième prélèvement. Des études complémentaires confirmeront ou infirmeront la faible pente de ces courbes dans les prélèvements suivants.

\section{CONCLUSION}

Comme nous l'avons déjà souligné toutes les espèces présentes à Saut Dalles ont été récoltées à Petit Saut. La zone de Saut Dalles ne semble donc pas comporter d'espèces endé- 
Tableau 5. - Comparaison du nombre d'individus capturés à Saut Dalles et à Petit Saut. Table 5. - Comparison of individual number captured in Saut Dalles and Petit Saut.

\begin{tabular}{|l|c|c|c|c|c|c|} 
& $\begin{array}{c}\text { Décembre } \\
1990\end{array}$ & $\begin{array}{c}\text { Juin } \\
1991\end{array}$ & $\begin{array}{c}\text { Août } \\
1991\end{array}$ & $\begin{array}{c}\text { Novembre } \\
1991\end{array}$ & $\begin{array}{c}\text { Janvier } \\
1992\end{array}$ & $\begin{array}{c}\text { Ensemble } \\
\text { des prélèvements }\end{array}$ \\
\hline Petit Saut & 226 & 131 & 147 & 48 & 140 & 692 \\
\hline Saut Dalles & 34 & 64 & 60 & 15 & 145 & 318 \\
\hline
\end{tabular}

$\mathrm{Ht}$ Sign Ht Sign Ht Sign Ht Sign Non sign Ht Sign

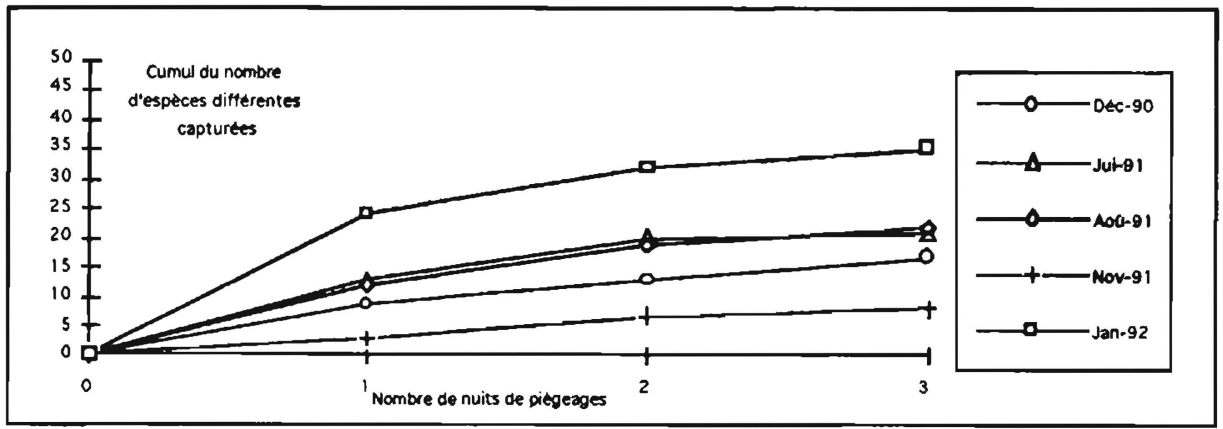

Fig. 1. - Evolution du nombre d'espèces capturées en fonction du nombre de piégeages effectués à Saut Dalles.

Fig. 1. - Quantitative evolution of night-light traps in view of obtaining representative sample of « in situ» fauna. Capture point: Saut Dalles.

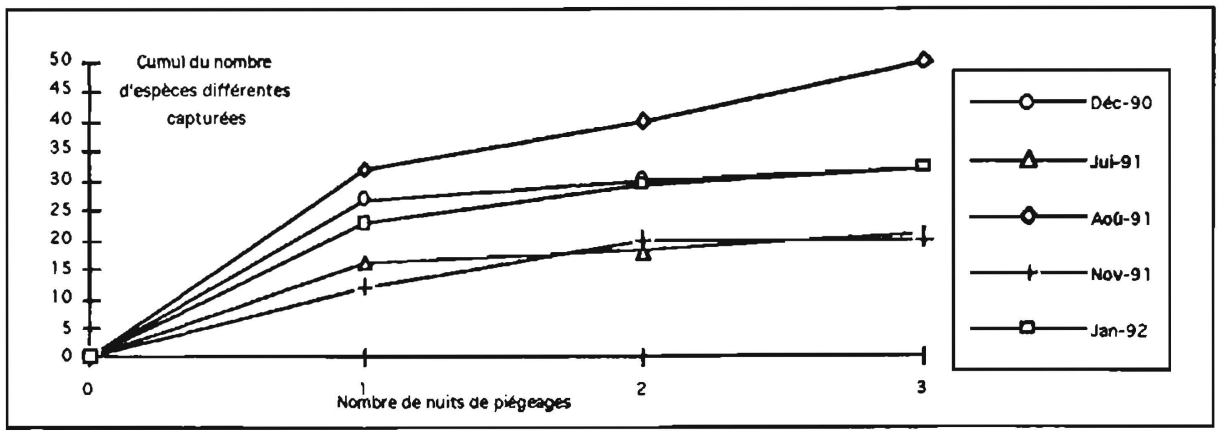

Fig. 2. - Evolution du nombre d'espèces capturées en fonction du nombre de piégeages effectués à Petit Saut.

Fig. 2. - Quantitative evolution of night-light traps in view of obtaining representative sample of " in situ" fauna. Capture point: Petit Saut. 
miques particulières aux marécages arbustifs. La comparaison entre Petit Saut et Saut Dalles montre que le peuplement de Sphingidae de Saut Dalles est moins riche quantitativement et qualitativement que celui de Petit Saut. Elle souligne l'aspect particulier des zones de forêts inondées. Les indices de Shannon et d'équitabilité montrent que les peuplements sont stables, aucune espèce n'a pullulé lors de ces 5 prélèvements. Pour chacun de ces prélèvements trois nuits de piégeage ont été nécessaires.

De nombreux auteurs (Habert et al., 1989; Owen 1972, Riveny et al., 1967, Robertson 1977 a et b) ont montré des différences importantes dans la phénologie des populations. A Petit Saut, une autre étude plus détaillée, menée en parallèle (Cerdan, 1992) a montré que certaines espèces semblent avoir une dynami- que propre, indépendante des saisons, d'autres sont bien liées aux saisons sèches ou humides. Mais pour la majorité des espèces il n'existe pas de différence significative dans les dates d'apparition des imagos.

Pour l'étude de tels peuplements les analyses statistiques montrent que les piégeages doivent couvrir au moins un cycle annuel. En effet, les différences ne sont pas significatives pour 2 des 5 prélèvements, de grandes variations peuvent apparaître d'une saison à l'autre. Le résultat final, pour l'année entière, est lui hautement significatif.

Dans la cuvette qui sera bientôt occupée par le lac de la retenue, l'essentiel de la superficie est couvert par la forêt primaire non inondable. Aussi l'impact sur les Sphingidae concerne davantage les peuplements les plus riches.

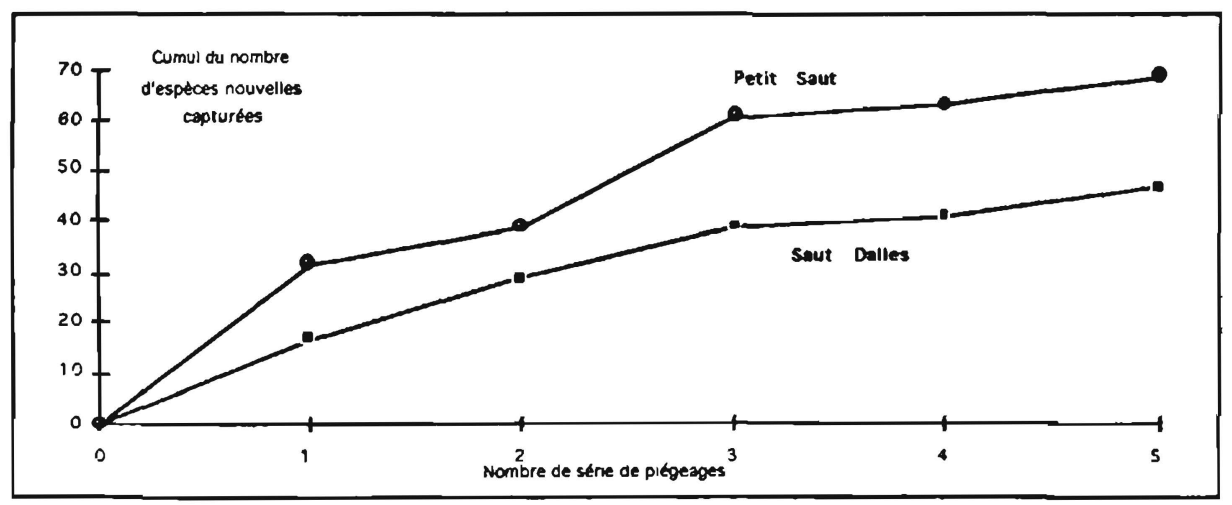

Fig. 3. - Estimation du nombre de prélèvements pour obtenir un échantillon représentatif de la faune en place.

Fig. 3. - Quantative estimation of prelevements in view of the obtantion of representative sample of "in situ" fauna. 
AUTEURS CITÉS

Blomberg O., Itamies J., \& Kuusela K., 1978. The influence of weather factor on Insect catches in traps equipped with different lamps in northern Finland. Ann. Ent. Fenn., 44, 56-62.

Cerdan P., 1991. Contribution à l'étude des arthropodes de Petit Saut au travers d'une famille de papillons: les Sphingidae. C.R. Com. Sc., J. Env., 79-82.

Cerdan P., 1992. Etude des arthropodes de Petit Saut au travers d'une famille de lépidoptères : les Sphingidae. Rapport EDF, $97 \mathrm{p}$.

Eisikowitch D. \& Galil G., 1971. Effect of wind on the pollination of Pancratium maritimum L. (Amaryllidaceae) by hawkmoths (Lep. Sphingidae). Jour. Anim. Ecol., 40, 673-678.

Fontaine J., 1982. Le piégeage lumineux, moyen d'approche de la faune entomologique d'un grand fleuve (Ephémèroptères en particulier). Bull. Soc. Linn. Lyon, 51, 81-89.

Habert W.A. \& Frankie G.W., 1989. A tropical hawkmoth community : Costa Rican dry forest Sphingidae - Biotropica, 21, 155-172.

Haxaire J., 1985: Le genre Xylophanes Hübner (1919) en Guyane française (Lepidoptera Sphingidae). Bull. Soc. Sci. Nat., 47, 21-27.

Haxaire J., 1986. La tribu des Smerinthini Hübner en Guyane française (Lepidoptera Sphingidae) Bull. Soc. Sci. Nat., $51,2-5$.

Haxaire J., 1987. Le genre Xylophanes Hübner (1919) en Guyane française. Quelques complements (Lepidoptera Sphingidae). Bull. Soc. Sci. Nat., 55, 15-19.

Haxaire J., 1988. La tribu des Sphingini (Latreille) en Guyane française (Lepidoptera Sphingidae) Bull. Soc. Sci. Nat., 59, 1-6.
Haxaire J., 1990. Le genre Manduca Hübner (1807) en Guyane française. Approche écologique et remarques systematiques (Lepidoptera Sphingidae). Bull. Soc. Sci. Nat., 55, 15-19.

Haxaire J., 1992. Les Aleuron Boisduval (18709) en Guyane française (Lepidoptera Sphingidae) Bull. Soc. Sci. Nat., 73, 1-16.

Haxaire J. \& Rasplus J.Y., 1986. Contribution à la connaissance des Sphingidae de Guyane française $1^{\text {er }}$ partie. Bull. Soc. ent. Fr., 91, 275-285.

Haxaire J. \& Rasplus J.Y., 1987. Contribution à la connaissance des Sphingidae de Guyane française $2^{\mathrm{e}}$ partie. Bull. Soc. ent. Fr., 92, 45-55.

Kislev M.E., Kraviz Z. \& Lorch J., 1972. A study if hawkmoth pollination by palynological analysis of the proboscis. Israel jour. Bot., 21, 57-75.

Linhart Y.B. \& Mendenhall J.A., 1977. Pollen dispersal by hawkmoths in Lindenis rivalis Benth. population in Belize. Biotropica, 9, 763-774.

Miller R.B., 1981. Hawkmoths and geographic patterns of floral variation in Aquilegia caerulea. Evolution, 35, 763-774.

Owen D.F., 1972. Species diversity in tropical Sphingidae and a systematic list of species collected in Sierra leone. Journal of Natural History, 6, 177-194.

Riveney E. \& Yathom S., 1967. Phenology of Sphingidae in Israel. Zeitschrift fur Angewandte Entomolog., 59, 384-392.

Robertson, 1977a. Reccords of insects taken at light in Tanziana $V$ : Seasonal changes in catches and effect of luna cycle on hawkmoths of the subfamily Asemanophornae (Lepidoptera : Sphingidae). Center for Overseas Pest research, Lond. Miscellaneous, 31, 1-14.

Robertson, 1977b. Records of insects taken at light in Tanziana VI: Seasonal changes in catches and effect of luna cycle on hawkmoths of the subfamily 
Semanophorinae (Lepidoptera: Sphingidae). Center for Overseas Pest research, Lond. Miscellaneous, 37, 120.

Tshernyshev W.B. \& Bogush P.P., 1973. Influences weather on flight of insects toward light, in central Asia. Moscow Iniv. Publ., 52, 700-701.

Usseglio-Polatera P. \& Auda Y., 1987. Influence des facteurs météorologiques sur les résultats de piégeage lumineux. Annls. Limnol., 23, 65-79. 